\title{
Microbiota mitochondrial metabolism
}

\begin{abstract}
The Microbiota is recognized by scientists around the world as a real organ of our body with billions of microbes at the center of biological and even neurological balance, for example the action of production of neuromodulators synthesized on site by the digestive mucosa and this flora. The intestine becomes an organ, a platform for regulating our metabolism and our body. It is also named:" the second brain", and near it, lives this flora that now proudly bears the name of microbiota. Based on the latest scientific findings, Dr. Richard Haddad explains the impact of the microbiota on our health and its impact on diseases such as diabetes, hypertension, obesity, etc. He creates the "Microbiotic Diet". For more than 40 years, Dr. Richard Haddad has been interested in weight gain and the mechanisms that lead to effective dieting. Specialist in nutrition, in the management of obesity and diabetes, he leads many conferences about this subject. The notion that emerges from all this work is, that the prebiotics fibers play an important and even essential role in the metabolism of our organism with a coordinated action with each cell via the mitochondria also of bacterial origin. He proposes a new term for this metabolic process:" the supply chain for health"
\end{abstract}

Volume 8 Issue 4 - 2018

Richard Haddad
Hospital Saint Louis Lariboisiere, France

Correspondence: Richard Haddad, Specialist, Hospital Saint Louis Lariboisiere, France, Email richardhaddad92@gmail.com

Received: June 21, 2018 | Published: August 07, 2018

Keywords: microbiota, probiotics, prebiotics, obesity, diabetes, second brain, gut, leaky gut, soluble fibers, microbiotic diet, the supply chain for health

Abbreviations: 3M, mitochondria microbiota and metabolism; $2 \mathrm{M}$, mitochondria microbiota

\section{Introduction}

The rate of obesity and diabetes has been growing steadily for many years, to combat this, Dr. Haddad has spent 40 years of professional practice looking for the ideal diet. The discovery of the role of Microbiota and the action of prebiotics has completely undermined the metabolic and dietetic approach of Nutrition. Approach often difficult to understand even for specialists. ${ }^{1}$

It is certain that the origin of life on earth has been bacterial for 4 billion and $1 / 2$ years. The persistence of billions of bacteria in our organism is for me the normal extension of this collaboration for the creation of life on earth. This advanced commensalism allowed the presence of a bacterial emissary in the cell itself, in the form of Mitochondria. And Mitochondria also have a bacterial origin. Microbiota and Mitochondria work in close collaboration for the survival of our organism. The deficiency of this microbial couple (by pesticides, heavy metals ...) is then at the origin of all or part of the diseases of our industrial period. Since a long time, people use fermented products: the probiotics.

\section{The probiotics}

a. Probiotic foods have been widely used, for thousands of years, to improve their health by all people depending on their type of diet.

b. Ginger beer gaseous, naturally fermented, probiotic drink appeared in England in the1800s.

c. Kefir This thick and pungent yogurt drink contains a wide variety of probiotic bacteria

d. Kimchi With a spicy flavor (garlic, salt, peppers, vinegar) is very popular in Korea

e. Kombucha fermented sweet black tea since 2000 years in China. f. Miso Thick soy paste plus a mushroom kojikin, very salty and very spicy.

g. Sauerkraut Fermented shredded cabbage, use cooled fresh form.

h. Yogurt Fermented dairy product with "live and active cultures"

i. Fortunately, the discovery of the prebiotics and their role on the health of the bacteria will allow an improvement and stimulation of all microbiota bacteria and consequently improvement of the health of every people

\section{The prebiotics}

The prebiotic foods are, now, going to be used more and more, The most used in the United States is inulin but poorly tolerated in high doses, other FOS and GOS are better supported at high therapeutic doses; And it is certain now that they interact with the bacteria of the intestine, the Microbiota, triggering the metabolic reactions that we will see. ${ }^{2}$ It is certain that the microbiota with prebiotics, but also stimulated by them, leads to a cascade of reactions, with formation of short chain fatty acid, and other metabolites : neurotransmitters that, with mitochondria are part of a metabolic process from the origin. This process provides each cell of our body with the minimum amount of nutrients. In detail, the main metabolites of the microbiota are: short chain fatty acids, urolithins and lactate. In particular, butyrate, which is produced by fermentation of non-digestible fibers by a number of different bacterial lines, and activates AMP Kinase. The microbial diversity enhances productivity, which improves mitochondriogenesis or Mitobioba for Zorov and co (microbiota / mitochondria relationship). The ultimate goal of this process: to give to the cell and to the mitochondria nutrients and energy. Here, I think, but the future will confirm, that energy efficiency with prebiotics is immeasurably higher than that of probiotic. ${ }^{3}$

\section{Conclusion}

The role of the Intestine has been better defined, and in fact its role as a waste receptacle passes second, leaving the role of 
Microbiota in the foreground with the action of billions of bacteria on our metabolism. But the discovery of soluble prebiotic fibers revolutionizes the approach of Nutrition. In fact, the soluble prebiotic fibers by their stimulating effect on the Microbiota and the liberated AG regulate our entire Metabolism. The European institutions in Brussels confirm this work and attribute two allegations "Health" to prebiotic fibers: one on food drives, and one on decrease in caries. In the Dietetic Diet "Microbiotic Diet" (www.regimeefficace.net) Dr. Haddad uses prebiotic fibers in Obesity and Diabetes An anomaly in this system, in this supply chain, leads to disease, cancer and death.

\section{Acknowledgments}

None.

\section{Conflicts of interest}

The author declares there is no conflict of interest.

\section{References}

1. Gibson GR, Hutkins R, Sanders ME, et al. Expert consensus document: The International Scientific Association for Probiotics and Prebiotics (ISAPP) consensus statement on the definition and scope of prebiotics. Nat Rev Gastroenterol Hepatol. 2017;14(8):491-502.

2. The quote Microbiology Food Microbiology Microbiota Nutrition. 2017

3. Franco-Obregón A, Gilbert JA. The Microbiome-Mitochondrion Connection: Common Ancestries, Common Mechanisms, Common Goals. mSystems. 2017;2(3):1. 